\title{
SISTEMA Y REALIZACION EN LA METRICA: BASES ANTIGUAS DE UNA DOCTRINA MODERNA
}

\begin{abstract}
The aim of this article is to show that principles such as the differences between Form and Structure or Rhythmic Form and Metric Form, so widely used in cont.mporary metrics studies, have already been clearly developed within the Rhythmics doctrine in Classical times, implicitly through the distinction between $\rho \cup \theta \mu \delta \varsigma$

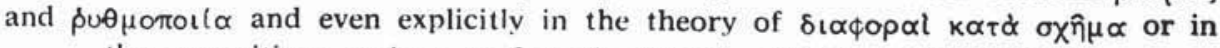

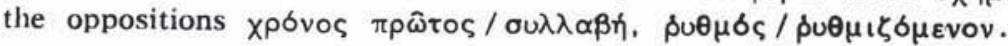

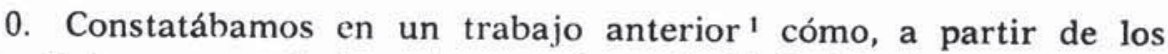
formalistas rusos y de los estructuralistas, la distinción dentro del fenómeno métrico entre el sistema, de un lado, y su realización, de otro, se ha ido luego precisando ${ }^{2}$ en varios niveles de funcionamiento y análisis que se pueden organizar del siguiente modo:

1 J. Luque Moreno, «Niveles de análisis en el lenguaje versificado», Homenaje al Prof. Rodriguez Adrados, Madrid, en prensa.

2 Cf., por ejemplo, con las naturales divergencias entre ellos, V. Erlich, E1 formalismo ruso, trad. J. Cabanes, Barcelona 1974, p. 306; R. Jakobson, «La lingüística y la poétican, en Th. A. Sebeok (ed.), Estilo del lenguaje, trad. A. M.* Gutiérrez Cabello, Madrid 1974, p. 149; T. Navarro Tomás, Métrica española, Madrid $1974_{5}$, pp. 27, 30 y 39, y Los poetas en sus versos, Barcelona 1973, p. 21; W. Kayser, Interpretación y andilisis de la obra literaria, trad. M.a D. Mouton y V. G.a Yebra, Madrid 1970, p. 315 ss.; H. N. Porter, "The early Greek Hexameter», YCS 12, 1951, pp. 2-62, esp. p. 8 ss.; S. Chatman, A Theory of Meter, La Haya 1965, p. 95 ss.; S. Mariner, "Hacia una métrica estructural", RSEL 1, 1971, p. 310 s.; M. HalleS. J. Keyser, "Chaucer and the study of Prosody», College English 28, 1966, pp. 187. $219=$ D. C. Freeman (ed.), Linguistics and Literary Style, Nueva York 1970; M. Halle, "On meter and prosody» en M. Bierwisch-K. E. Heidolph (edd.), Progress in Linguistics, La Haya 1970, p. 64; J. Roubaud, "Mètre et vers», Poétique 7, 1971, pp. 366-387; W. S. Allen, Accent and Rhythm, Cambridge 1973, p. 103 ss.; P. Kiparsky, "Stress, Syntax, and Meter», Language 51, 1975, pp. 576-616; M. E. Loots, Metrical Myths, La Haya 1980, pp. 15, 192. 
Dos primeros niveles dentro del "sistema" ("verse design" de Jakobson o "meter" de Halle) que suponen dos grados distintos de abstracción:

A) Forma o instancia más abstracta y universal, que define el verso en su sentido más general. Por ejemplo, «hexámetro dactílico»:

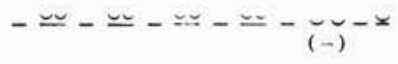

B) EsTRUCTURA, una de las variantes permitidas por la FORMA:

Vienen luego otros dos niveles que representan en distintos grados y maneras la realización del sistema:

C) Composición (que se corresponde con "verse instance», "mapping", "actualization", en la terminología de Jakobson o de Halle) o cumplimentación de la ESTRUCTURA a base de términos de realización lingüística:

arma uirumque cano Troiae qui primus ab oris

D) ReALización o ejecución llevada a cabo por parte de una persona roncreta en un momento y circunstancias concretos.

Suponen estos niveles distintos grados de abstracción y su funcionalidad reside en la creación y mantenimiento de una tensión constante entre lo uniforme y lo diverso, entre lo unitario y lo múltiple, entre lo regular y lo variado ${ }^{3}$.

Ante todo ello nos situábamos entonces con la intención de hacer varias consideraciones sobre las posibilidades que para la métrica latina se abren con la distinción de dichos planos funcionales y tratando a la vez de aportar algunas precisiones a dicho esquema de niveles desde el campo de la métrica latina.

Lógicas limitaciones de espacio no nos permitieron allí desarrollar todos los aspectos con el debido detenimiento. $\mathrm{Y}$ es ésta la razón por la que hoy volvemos sobre el tema con la intención de completar lo que allí quedó expuesto; más en concreto, de aportar algunos datos acerca de lo que los antiguos tratadistas de métrica y, sobre todo, de rítmica pensaban acerca de algunas de estas cuestiones. Pues sorprendentemente algunas de ellas que hoy se tienen por un logro decisivo de

3 Cf. Allen, op. cit., p. 105 ss.; R. Fowler, "Prose rhythm and meter", en Freeman, op. cit., p. 345 ss.; Kiparsky, op. cit., p. 583 ss. 
los modernos métodos lingüísticos en el terreno de la métrica creemos encontrarlas ya explícita o implícitamente definidas en los escritos de época griega y romana, sobre todo si remontamos a los tratadistas de rítmica de época clásica, así como a los primeros metricólogos, aunque sólo sea en la medida en que éstos reflejan sin adulterar la doctrina de aquéllos.

1. La primera consideración que hacíamos entonces era la de la conveniencia, y casi necesidad por su alto rendimiento metodológico, de establecer una nueva distinción a nivel de FORMA: diferenciar dos niveles, uno más abstracto y general que llamábamos FORMA EXTRALINGǗSTICA O FORMA RITMICA y otro, FORMA LINGǗSTICA, que no sería otra cosa que la plasmación de la primera dentro del campo del lenguaje y con elementos de él tomados.

Ejemplificando con el hexámetro dactílico, la forma extralingüística sería

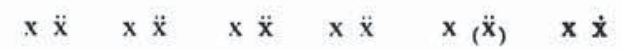

en donde con $/ \mathrm{x} / \mathrm{y}$ con /./ representamos dos elementos distintos cualesquiera, organizados según una convención rítmica determinada.

Dicha forma rítmica puede materializarse de mil maneras, tantas como equivalencias puedan tener $/ \mathrm{x} / \mathrm{y} / . /$ en el ámbito de la experiencia humana: colores, movimientos, sonidos diferentes en timbre, en intensidad, en duración, en articulación, etc.

La forma lingiiística es sólo una de esas múltiples posibilidades, llevada a cabo convencionalmente mediante la elección convencional de unos elementos del sistema lingüístico ${ }^{4}$.

4 De suyo, para ser más exactos, habria que distinguir, entre lo que denominamos FORMA EXTRALINGUÍSTICA y la FORMA MÉTRICA, un plano intermedio, considerando:

a) Forma eXtralingUf́stica, a la que también se la nodría denominar FoRMa RftMICA, entendiendo aquí ritmo como patrón general abstracto, del cual el metro sería luego una concreción.

b) Distintas posibilidades generales de concreción: visuales («forma visual»). motrices ("forma motriz.n), acústicas ("forma acústica»).

c) FORMA Métrica, que sólo es un caso más de la forma acústica o de la forma acústico-motriz (si se quieren recoger las doctrinas motrices sobre la silaba o puntos de vista como los de V. Zirmunskij, Introduction to Metrics, trad. C. F. Brown, La Haya 1966, pp. 19 y 89, o D. Abercrombie, "A phonetician's view of verse structure», en Studies in Phonetics \& Linguistics, Londres 1965, p. 16, o Allen, op cit., p. 100).

Por supuesto que, ante las anteriores distinciones, la realización escrita de la FORMA MÉTRICA es normalmente sólo una segunda convención para representar dicha forma métrica, que es de índole acústica o acústico-motriz. Sólo en casos espe- 
1.1. Pues bien, con esta distinción no se hace más que recoger uno de los logros más decisivos de la doctrina rítmica griega, el alcanzado

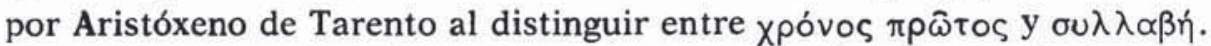

Es posible que en épocas anteriores a Aristóxeno, cuando las tres artes musicales (orquéstica, música y poética) formaban un todo unitario en el que la danza y sobre todo la melodía y las palabras eran plenamente solidarias, la duración de los tonos de aquélla se ajustase y se rigiera exclusivamente por la duración de las sílabas ${ }^{5}$. En ese caso ritmo y metro (en el sentido actual de los términos) habrían sido lo mismo y se justificaría no ya sólo el menor desarrollo que en general parece haber tenido en la Antigüedad la teoría rítmica frente a la harmónica, sino, y sobre todo, el hecho de que, por lo que se puede vislumbrar a través de la fuerte penuria de datos, la rítmica anterior a Aristóxeno aparezca absolutamente ligada al ritmo del habla.

Como se deduce de algunos pasajes de Platón ${ }^{6}$, Aristóteles ${ }^{7}$ o Aris-

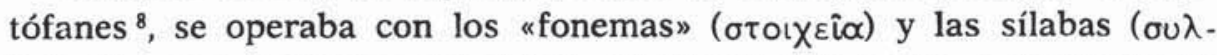
$\lambda \propto \beta \propto())$ como elementos del ritmo, sin llegar, por tanto, a desligarlo de

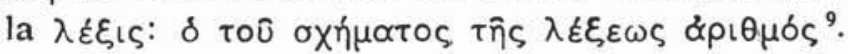

En la observación y análisis del ritmo se había partido originariamente del movimiento del cuerpo y como unidad de medida había

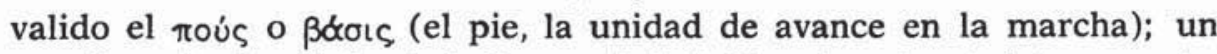
estudio más detallado de la lengua habría llevado a los teóricos a establecer la sílaba ${ }^{10}$ como unidad mínima, en el supuesto además de que

ciales podría entenderse como un artificio lingüístico-visual. Cf. sobre el particular T. Navarro, Métrica..., p. 35, n. 3 .

5 No se puede demostrar, pero es muy probable, que en tiempos de Safo, de Alceo y de la primera lírica coral el ritmo del melos no fuese algo independiente del ritmo de la $\lambda \hat{\varepsilon} \xi$ is.

6 Crat. $424 \mathrm{c}$.

7 Rhet. 1408 b.

8 Nubes 638 ss.

9 Aristóteles, Rhet. 1408 b.

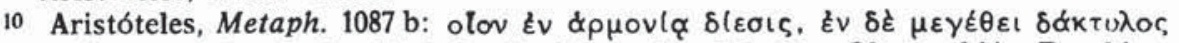

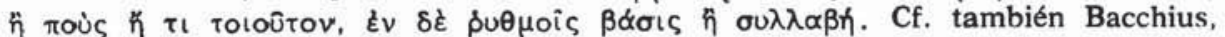

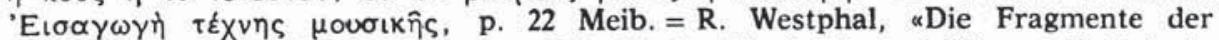
Rhythmiker...n, p. 46, suplemento a Griechische Rhythmik und Harmonik, Leipzig

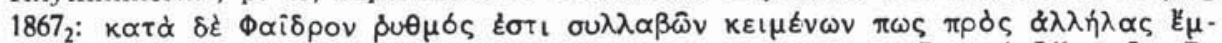
$\mu \varepsilon \tau$ роৎ $\theta$ f́oıs; Varrón en (Sergii) Explanationum in artem Donati Liber I, «De accentibus», GLK IV 533, 12 ss.: tempus ad rythmicos pertinet, syllaba ad metricos. Inter rythmicos et metricos dissentio nonnulla est, quod rythmici in uersu longitudine uocis tempora metiuntur et huius mensurae modulum faciunt tempus breuissimum, in quo cum quae syllaba enuntiata sit, breuem uocari; metrici autem uersuum mensuram syllabis comprehendunt et huius modulum syllabam breuem arbitrantur, tempus autem breuissimum intellegi, quod enuntiationem breuissimae syllabae cohaerens adaequauerint. Itaque rythmici temporibus syllabas, metrici tempora syllabis faciunt. 
rítmicamente una sílaba breve valía la mitad que una larga y de que dos o más sílabas forman un roús.

Ahora bien, como se puede deducir por múltiples indicios ", la propia realidad del habla debía demostrar que las ecuaciones ${ }_{-}=\cup 0$ - / $=2 / 1$ en modo alguno eran exactas y que, por tanto, no se podía basar en ellas la medida o determinación del decurso temporal. Si a ello añadimos el progresivo desarrollo del $\mu \varepsilon \lambda$ o $\mathrm{y}$ los primeros pasos de una música instrumental independiente del texto, se comprenderá enseguida que conjuntamente con la escisión e independización de las tres artes rítmicas o musicales se produzcan dos fenómenos distantes en apariencia, pero intimamente relacionados: en el plano práctico el que se empiece a sentir la necesidad de introducir en los textos, en las "partituras", unas marcas que precisen la duración de los distintos tonos de la melodía; en el plano teórico, que se alcance una definición del ritmo como algo independiente del "metro" del texto, o sea, por encima de e independiente de la $\lambda \varepsilon \xi$ เs, de las sílabas.

De ambos fenómenos el más antiguo testimonio explícito que poseemos es el de Aristóxeno de Tarento.

Aristóxeno parece haber sido el primero en pronunciarse en contra de sus predecesores al establecer como una de las premisas básicas de su rítmica el principio de que las sílabas, al no ser una magnitud duracional fija, no pueden servir como unidad de medida del ritmo cuyo substrato primario es el tiempo:

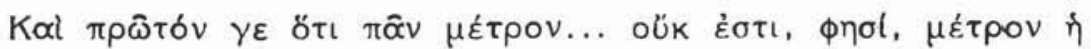

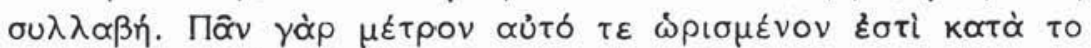

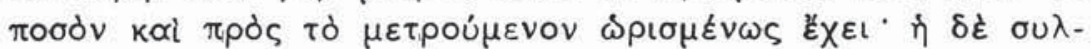

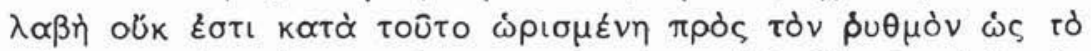

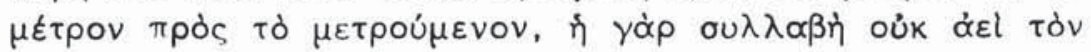

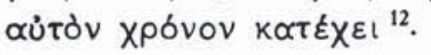

Quidam autem non pedem metrum esse uolunt, sed syllabam, quod hac ipsum quoque pedem metiamur et quod finita esse mensura debeat, pedes autem in uersu uarientur. Alii rursus nec pedem nec syllabam metrum putant esse dicendum, sed tempus, quia omne metrum in eo quod metimur numero finitum est... ${ }^{13}$.

11 Cf., por ejemplo, A. J. Neubecker, Altgriechische Musik, Darmstadt 1977. p. 122.

12 M. Pselo, Proslambunomena, ed. R. Westphal, "Die fragmente...», p. 18 ss.

13 Mario Victorino, Ars Grammatica I, GLK VI 51, 29-32. 
El ritmo es una ordenación de magnitudes temporales: "Eotı $\delta \dot{\varepsilon} \delta$

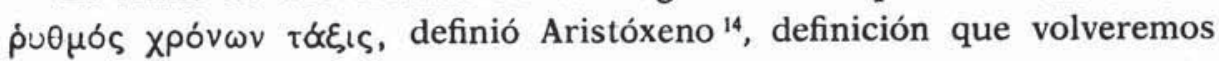

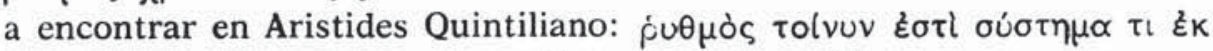

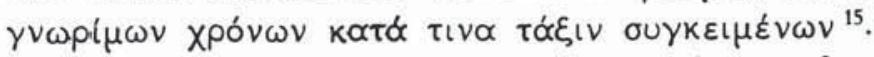

El ritmo es una cierta estructura o sistema formado a base de una determinada ordenación o disposición de tiempos perceptibles. Su unidad de medida es por ello una magnitud temporal, no divisible, aunque sí multiplicable: el $\chi \rho \delta$ vos $\pi \rho \hat{\tau} \tau$,, al cual se agregan como múltiplos

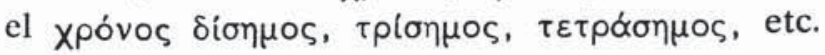

1.2. Ello no quiere, sin embargo, decir que el $\chi p o ́ v o s ~ \pi p \hat{\tau} \tau o \varsigma$ sea una magnitud duracional fija. Vale hasta cierto punto para él lo que

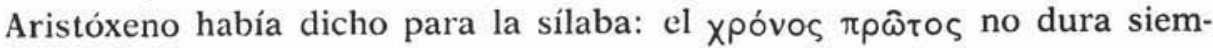
pre lo mismo (oủk ácl aủTóv Xpóvov $\kappa \alpha \tau \dot{\varepsilon} \chi \varepsilon \iota$ ) ya que puede ser más

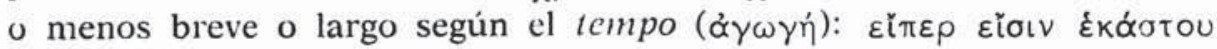

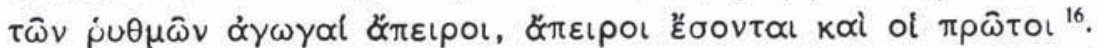

Ahora bien, el que estos xpóvol $\pi \rho \hat{\omega} \tau o l$ no tengan una duración fija no es obstáculo para que sirvan de medida. Cada ritmo que se ofrece al receptor como medible tiene una $\alpha \gamma \omega \gamma \eta \dot{n}$, un tempo concreto. Por

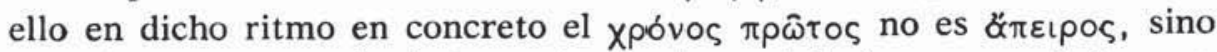
que tiene una magnitud determinada, lo cual lo hace apropiado para

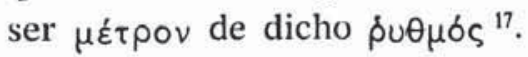

1.3. Se entra de este modo en el problema de la isocronía / anisocronía en las unidades rítmicas y métricas.

Decíamos en nuestro escrito anterior que por la vía de la distinción entre forma rítmica y forma métrica se podía empezar a clarificar el problema, asignando a aquélla la isocronía y a ésta la anisocronía.

No porque pensemos que todas las formas rítmicas, extralingüísticas, sean isocrónicas o que no puedan existir en el ritmo del lenguaje formas isocrónicas (en la medida en que la ejecución humana lo permita), sino por otra serie de cuestiones que merece la pena intentar dilucidar,

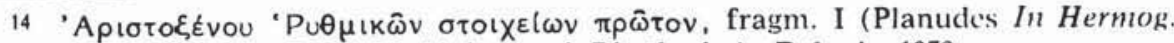
id. V 454 W), ed. G. B. Pighi (Aristoxeni Rhythmica), Bolonia 1959.

15 De Musica I, xiii, ed. R. P. Winnington-Ingram, Leipzig 1963.

16 Aristóxeno, Пepi toû $\pi \rho \omega$ tou xpóvou (Porphyr. ad Ptol., p. 255), ed. Westphal, "Die Fragmente...", p. 15.

17 No obstante todo lo anterior, parece demostrado (cf., por ejemplo, Th. Georgiades, Die griechische Rhythmus, Hamburgo 1949) que, a excepción de ciertas formas especiales, como marchas, canciones de remeros, etc., el ritmo de la música griega, como el de la romana y el de la Alta Edad Media, no era tampoco algo tan estricto y sometido a un compás regular como el de la musica mensurata posterior.
} 
máxime cuando para ello nos aportan datos sustanciales los propios escritos de los tratadistas de rítmica antiguos.

Ante todo hay que decir que, al asignar la isocronía a la forma rítmica, no estamos usando el término "isócrono" en el sentido de que esta forma rítmica tenga que estar organizada a base de unidades o intervalos iguales o regulares, sino en el de que dichos intervalos, convencionales ${ }^{18}$, se conciben como idealmente isócronos cada vez que aparecen en su convencional retorno. Aceptamos con Loots que «if not on the production level, isochronous intervals derive some support on the perception level in the sense that subjects tend to under- and overestimate actual intervals in a tendency towards isochrony" ${ }^{19}$. Y por

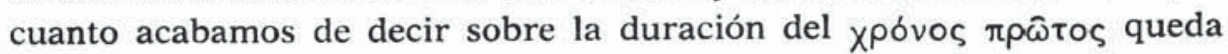
claro que no contradicen dicha tendencia cosas tan naturales y conocidas como los cambios de tempo (incluso en la música occidental moderna, sometida a un compás fijo) dentro de un mismo ritmo binario, ternario, etc.: las blancas de un prestissimo pueden durar menos que las negras de un largo sin que el ritmo deje de ser el mismo (binario, ternario, etc.), con tal que tanto en uno como en otro tempo se mantenga la convención _ = $\smile$. Y lo propio, y aún más, en los rallentandi producidos a lo largo de un mismo patrón rítmico.

Evidentemente casos como los mencionados hacen ver el error que supone concebir una duración física absolutamente fija para una blanca o una negra en la música o, a fortiori, para una sílaba larga o breve en el lenguaje.

Pero no es en este sentido en el que aquí estamos hablando de isocronía. Cuando hablamos de isocronía nos movemos exclusivamente en el plano de la pura abstracción, en el plano del cálculo de las relaciones convencionalmente establecidas entre los elementos de la convención rítmica.

1.4. Se apunta además en todo lo anterior la distinción entre verso recitado y verso cantado, entre habla y canto, entre métrica y música, diferencia a la que tampoco fueron ajenos los antiguos rítmicos, máxime cuando una producción como la de Aristóxeno se escribió en un momento en que las tres artes musicales empezaban ya a emanciparse y a recorrer cada una su propio sendero, rompiéndose el todo unitario en el que hasta hacía poco habían estado integradas.

18 S. Mariner, "Carácter convencional del ritmo", Coloquios de Historia y estructura de la obra literaria, Madrid 1971, pp. 89-96.

19 Loots, op. cit., p. 113. 


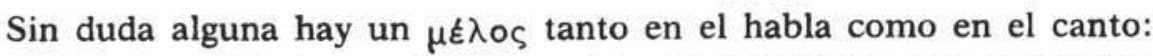
en ambos casos (tanto aquélla como éste pueden haber sido desarrollados a partir de un núcleo originario común) se trata de sonidos, que no son otra cosa que unos timbres, unas intensidades, unos tonos y unas duraciones. La diferencia está en que el movimiento de la voz

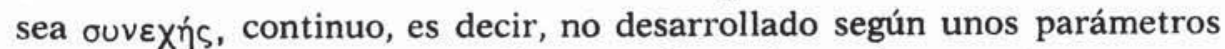

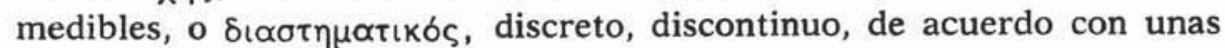
magnitudes tonales, de intensidad y de duración fijas $\mathrm{y}$, por tanto, medibles ${ }^{20}$.

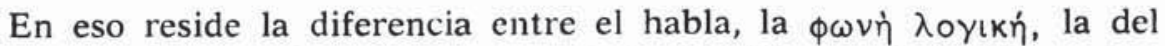

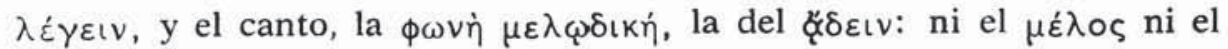

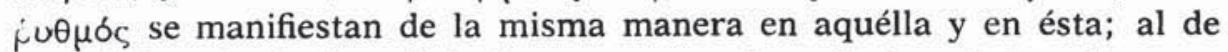
esta última lo designa Aristóxeno como "ritmo de la música" ( $\varepsilon v \mu \circ v$ -

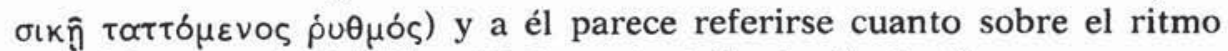
se habla a partir del libro II de su tratado de rítmica ${ }^{21}$.

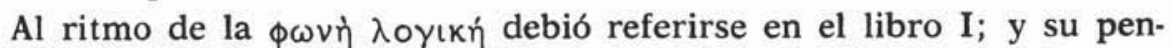
samiento sobre el particular se puede rastrear a través de algunos pasajes de la Primera Harmónica ${ }^{22}$ y de algún fragmento de Pselo que parece remontar a la Rítmica de Aristóxeno.

En el lenguaje hablado las sílabas son tiempos perceptibles, pero

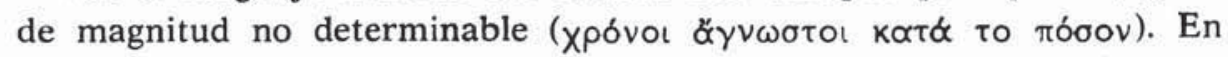
cambio, en el lenguaje cantado, con las salvedades hechas anteriormen$t^{23}$, las sílabas (y los tonos en general en la música) tienen una dura-

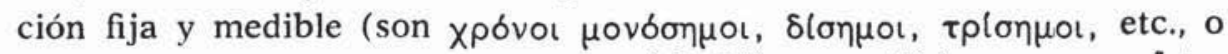

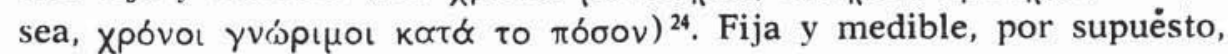
dentro de un determinado tempo o $\alpha \gamma \omega \gamma \eta \dot{n}$.

La distinción de dos duraciones silábicas, lingüística y métricamente pertinentes, - / u, no es una distinción estrictamente física (aunque tenga, por supuesto, cierta base física); ni siquiera es de la misma

20 «En un sentido las convenciones de la música serian un afinamiento, al tiempo que aumento en grados melódicos, de las músicas lingüísticas de la frasen, A. García Calvo, Del lenguaje, Madrid 1979, p. 131.

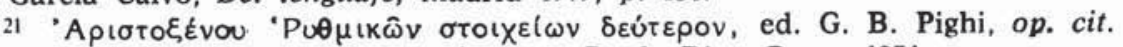

22 Aristoxeni elementa harmonica, rec. R. da Rios, Roma 1954.

23 Cf. nota 17 y A. W. de Groot, «Der Rythmus», Neophilologus 17, 1932, p. 83 ss.

24 Sin que ello quiera decir que no se den o que no se perciban diferencias de duración entre las sílabas del lenguaje hablado; evidentemente las hay, como también las hay de tono y de intensidad (máxime en una lengua en que dichas diferencias eran relevantes), pero no medidas o medibles más o menos exactamente como en el canto y en la música en general. Cf. R. Westphal-R. Gleditsch, Allgemeine Theorie der griechischen Metrik, Leipzig 1887, p. 9 ss.; H. Weil, recensión de la obra anterior en su segunda edición, Journal des savants, febrero de 1894, p. 113 ss. 
naturaleza y precisión temporal que la distinción $d / d$ dentro de la música "medida" (musica mensurata desde el siglo XII) occidental, pero tanto aquélla como ésta se basan sobre la misma forma rítmica, idealmente exacta, $2 / 1$.

La convención métrica - = $\checkmark$ no tiene naturaleza duracional, física, como la tiene, por ejemplo, en música la ecuación $d=d d$ o $d=d \downarrow$ ? pero se sustenta, al igual que ésta, sobre la forma rítmica más general $2=1+1$.

Una forma métrica como _ _ no tiene por qué ajustarse exactamente al esquema de duraciones de la forma musical $d d d$, pero se apoya, al igual que esta última, sobre la misma forma ideal, exacta, 21 1. En un ritmo lingüístico dactílico los dáctilos no tienen por qué ser todos iguales unos a otros en duración, pero están todos unificados por la anterior forma ideal, exactamente isócrona 211 .

La duración física de un hexámetro dactílico es con toda probabilidad distinta de la del que le precede y del que le sigue (y no ya sólo por el número de sílabas, sino por las múltiples posibilidades de sílabas distintas en volumen físico, etc.). Sin embargo, a todos esos hexámetros los unifica y sustenta una misma forma rítmica ideal.

Es así como la forma rítmica se constituye en patrón absoluto, idealmente isócrono, que subyace y unifica la forma rítmica del lenguaje versificado (forma métrica) o la de la música, representando estas últimas un grado menor o mayor, respectivamente, de aproximación a aquélla.

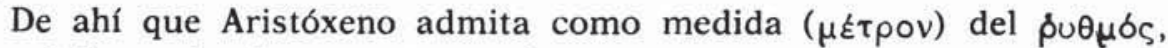

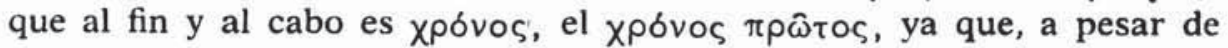
ser en teoría đ̌́ $\pi \varepsilon$ ipos, o sea, sin una duración fija, en la práctica, es decir, en un determinado tempo o $\measuredangle \gamma \omega \gamma \eta$, deja de serlo y resulta apropiado como medida abstracta para una magnitud abstracta.

De ahí también que Aristóxeno, en cambio, niegue a la sílaba la capacidad de ser unidad de medida, a pesar de que en la praxis, o sea, a nivel de forma métrica, la sílaba breve coincida con (o mejor, sea la

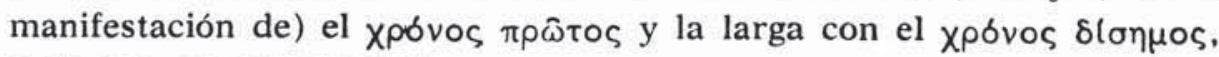
y de que, en consecuencia, $\ldots=\ldots$.

Aristóxeno tenía muy claro que la duración de las sílabas, las del lenguaje hablado, las del verso recitado, no es fija. Sin duda remonta a él la afirmación tan repetida por los tratadistas de música y rítmica posteriores, incluso por algunos metricólogos, de que la sílaba breve no dura siempre un tiempo, al igual que la larga no dura siempre dos. 
1.5. Mas con el anterior reconocimiento de la forma rítmica como instancia más abstracta a la que referir la forma métrica y con las diferencias establecidas entre canto y recitado estamos haciéndonos eco de otra de las distinciones fundamentales en la rítmica de Aristóxeno:

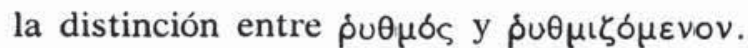

Discípulo destacadísimo de Aristóteles, Aristóxeno reproduce en la rítmica la distinción $\varepsilon i \delta \circ \varsigma / \measuredangle \lambda \eta$ del maestro.

El ritmo ( $\dot{0} \cup \mu \mu \delta \varsigma)$ es una forma abstracta $(\varepsilon \mid \delta \circ \varsigma)$, producto del espíritu humano, que la estampa sobre una materia ( $(\lambda \eta)$ susceptible de

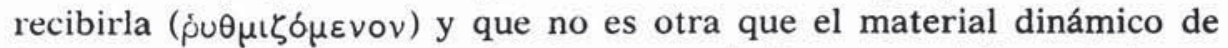
las artes musicales (o rítmicas), o sea, de las artes del movimiento, que se desarrollan en el tiempo.

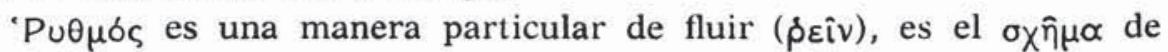
algo que se mueve: $\tau \hat{\eta} \varsigma \kappa \iota \nu \eta ́ \sigma \varepsilon \omega \varsigma ~ \tau \nless \alpha \xi ı \varsigma^{25}$. Precisamente teniendo en cuenta que $\dot{p} \hat{\imath} v$ es el predicado normal de la naturaleza y de las cosas en la filosofía jónica desde Heráclito, es como se comprende el sentido exacto de este término que encierra en si todo un largo proceso de reflexión y que ya en el pasaje citado se encuentra con las connotaciones de medida, de patrón, de orden, en la praxis de las artes musicales ${ }^{26}$.

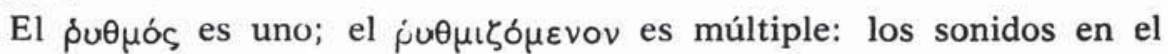

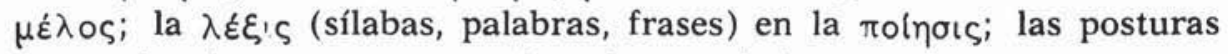

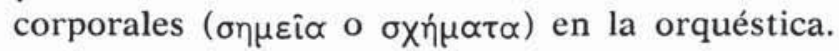

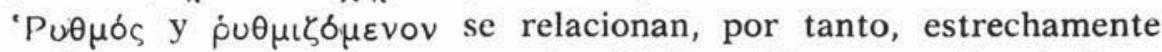
como forma y materia, pero son algo distinto. No se concibe el ritmo como una idea con existencia propia, a la manera en que podría haberlo hecho Platón; el ritmo sin el rhythmizómenon no puede tener realidad ninguna, sino que se manifiesta en cualquiera de las tres modalidades de este último, mediante una determinada ordenación de las partes: en la $\lambda \dot{\varepsilon} \xi$ เৎ a base de las letras, las sílabas, las palabras, etc. ( $\gamma \rho \alpha \mu \alpha \mu \alpha \sigma$ เ

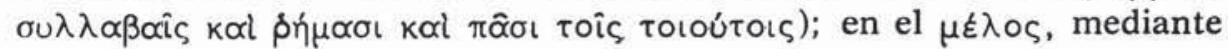

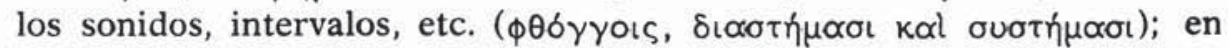
la danza a través de los movimientos y posturas del cuerpo (on $\mu \varepsilon$ íoı

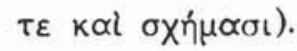

1.6. El ritmo, tal como lo concibió Aristóxeno, es, pues, el principio que informa las artes musicales, o sea, las artes cuya esencia reside

25 Platón, Leg. 665 a.

26 E. Benveniste, "La notion de rythme dans son expression linguistique», en Problèmes de linguistique générale, París 1966, pp. 327-335. Cf. también, por ejem. plo, W. Christ, Metrik der Griechen und Römer, Leipzig 1879, p. 1. 
en el movimiento y en la ordenación de las partes del tiempo, las

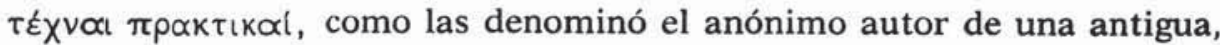
probablemente peripatética, ordenación de las artes ${ }^{27}$.

Es desde esta perspectiva como hay que entender la relación ritmo / metro cuando se define éste como una especie de aquél ${ }^{28}$.

Es también desde esta perspectiva como se justifica y explica nuestra propuesta de distinguir entre forma rítmica y forma métrica, concibiendo esta segunda como una especie o concreción de aquélla.

Se trata de dos planos que históricamente, a nivel de la música y de la métrica grecolatina, mantienen entre sí una muy estrecha dependencia, lo cual constituye sin duda una razón más a favor de la utilidad de su distinción.

Se trata de dos planos que a partir de Aristóxeno quedaron claramente definidos y fueron mantenidos en el mundo antiguo por los teóricos de la rítmica e incluso por los metricólogos, al menos por los que se mantenían más próximos a las doctrinas de los primeros. Será solamente en épocas mucho más cercanas a nosotros cuando los metricólogos, desconectados de las auténticas raíces rítmico-musicales de su disciplina por atender a una poesía casi exclusivamente recitada, por pesar sobre ellos su condición de gramáticos $\mathrm{y}$, especialmente, por la presión de una métrica casi exclusivamente gráfica y filológica, olviden esta perspectiva y se coloquen en otra mucho más miope, volviendo al principio de la medida de las sílabas, es decir, no levantando los ojos en el verso por encima de la forma métrica.

2. Mas no es sólo la distinción entre forma rítmica y forma métrica la que puede sustentarse sobre principios doctrinales establecidos ya en la Antigüedad. Existen también en la antigua doctrina sobre el ritmo elementos que permiten reconocer en ella algo muy similar a la distinción dentro del sistema de los dos niveles (FORMA y ESTRUCTURA) de que hablábamos al principio.

El ritmo, según se desprende de cuanto acabamos de decir, quedó ya definido para Aristóxeno y sus sucesores como algo abstracto, como una instancia superior que sustenta y garantiza la forma de cada una de las artes musicales a base de una serie de patrones que sobre el

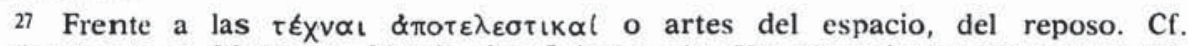
A. Rossbach-R. Westphal, Metrik der Griechen im Vereine mit den übrigen musi. schen Künsten I, Leipzig $1867_{2}$, p. 3 ss.

$28 \mathrm{Cf}$, por ejemplo, S. Agustín, De musica III 1: omne metrum etiam rhythmus, non omnis rhythmus etiam uersus; item omnis uersus et metrum sit, non omne metrum etiam uersus. Chatman, op. cit., p. 12: «meter is a species of rhythm», o p. 29: "meter is basically linguistically determined 'secondary rhythm'». 
material motor y temporal con que dichas artes operan son impuestos por una especie de tendencia natural del hombre a la ordenación y medida de ese tiempo y de ese movimiento.

Si como unidad mínima de medida de dichas magnitudes quedó ya establecida el xpóvos $\pi \rho \omega \hat{\tau} \circ \varsigma$, no hay que olvidar que se trata de eso, de una unidad de medida, no de una unidad rítmica: un solo tiempo no puede constituir ritmo, si no se pone en relación al menos con otro:

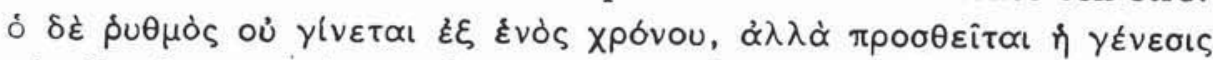

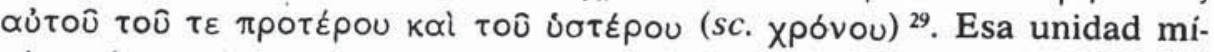
nima de producción y percepción del ritmo es el roús, término que en la rítmica de Aristóxeno designa tanto nuestro "pie» de la métrica como nuestro "compás» de la música ${ }^{30}$.

El pie es, por tanto, la unidad mínima rítmicamente significativa tanto para el que produce el ritmo como para el que lo percibe: $\Phi$

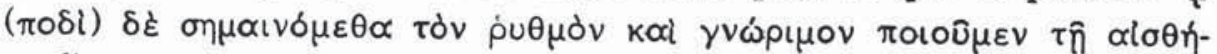

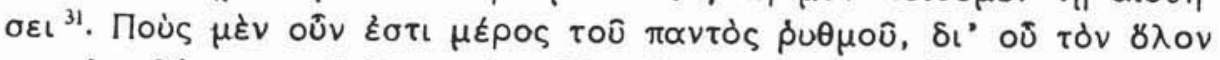

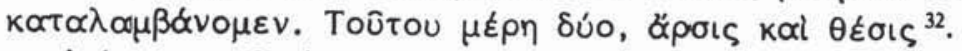

Así, pues, el pie o compás es básicamente una magnitud ( $\mu \varepsilon \gamma \varepsilon \theta \circ \varsigma$ ) temporal constituida por dos partes o, si se prefiere, por dos magnitudes temporales que se relacionan entre sí según un determinado $\lambda \delta \gamma \circ \varsigma$ (ratio). Es ese $\lambda \delta \gamma \circ \varsigma_{S}$ el que lo define y el que, en consecuencia, determina su $\gamma \varepsilon \dot{\varepsilon} \circ \varsigma^{33}$.

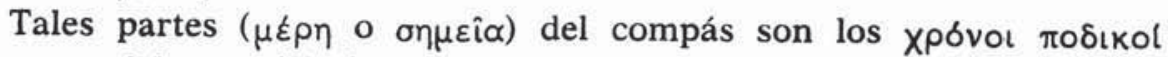
(tiempos del compás), formas o patrones abstractos que son marcados (оๆ $\mu \varepsilon \hat{i} \alpha)$ mental o físicamente por el que produce (compone, dirige o ejecuta) el ritmo y sentidos por el que lo percibe.

Pertenecen, por tanto, dichos $\chi \rho \delta$ vo $\pi \circ \delta ı$ ol exclusivamente a la forma rítmico-métrica o rítmico-musical y se mantienen, al igual que la unidad pie / compás, a nivel de la pura abstracción.

Se distinguen por ello netamente de los tiempos reales, físicos, que van dando cuerpo en cada caso a dichos patrones abstractos: estos

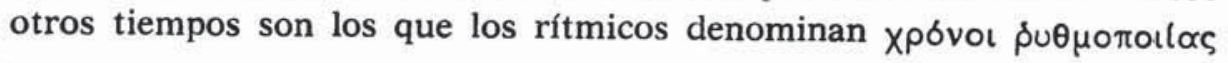

29 Pselo, $\S 4$, un pasaje que remonta probablemente al comienzo del libro se-

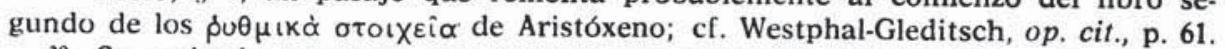

30 Se entiende, por supuesto, que se está hablando del compás o pie simples,

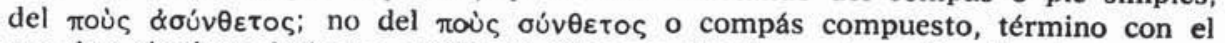
que los rítmicos designan entidades diversas, desde nuestra "dipodia» hasta nuestro "colon" e, incluso, nuestro "verso".

31 Aristóxeno, Elementa rhythmica II 288.

32 Aristides Quintiliano, De musica I, xiv.

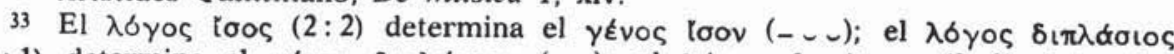

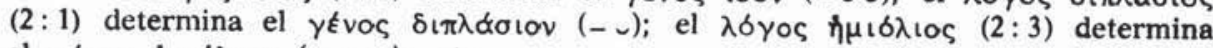

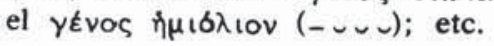




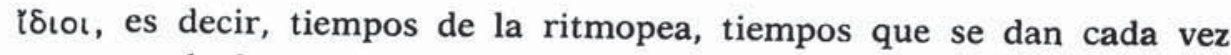
que una de las anteriores formas abstractas toma cuerpo a base de elementos de un $\delta \cup \theta \mu \zeta \zeta{ } \mu \varepsilon v o v$ concreto (del habla, del canto, de la danza).

Distinguen, pues, Aristóxeno y los rítmicos entre ritmo $(\rho \cup \theta \mu \delta \varsigma)$ y ritmopea $(\dot{\rho} \cup \theta \mu \sigma o r l \alpha)$ o realización de aquella estructura abstracta rítmica mediante elementos de un $\hat{\rho} \theta \theta \mu \zeta \delta \mu \varepsilon v o v$.

Por ejemplo, un troqueo no es más que un concepto abstracto, una

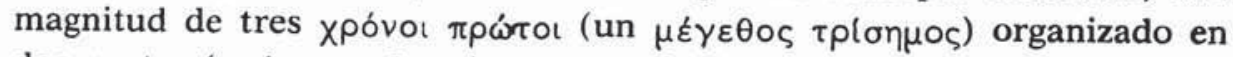

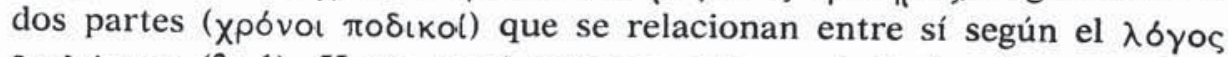
$\delta ı \pi \lambda$ đ́oı $(2: 1)$. Hasta aquí estamos sólo a nivel de ritmo o, si se quiere, incluso de "forma métrica, musical, etc.».

Ahora bien, dicha forma abstracta puede realizarse, por ejemplo, en el terreno de la métrica, a base de una sílaba larga y una sílaba breve $(-\cup)$ o a base de tres sílabas breves ( $\checkmark \cup$, dos en la primera parte y una en la segunda) o incluso, según los antiguos rítmicos, refiriéndose, por supuesto, a la poesía cantada, a base de una sola sílaba de tres tiempos ( ᄂ ). En el terreno de la música, y utilizando nuestra notación actual, la forma trocaica puede realizarse a base de $\rfloor \mathcal{L} \Lambda \mathcal{S}$ od.

Pues bien, a los tiempos de esta segunda instancia (física, en el terreno de un $\hat{\rho} \cup \theta \mu \zeta \delta \mu \varepsilon v o v$ específico) es a los que los rítmicos griegos

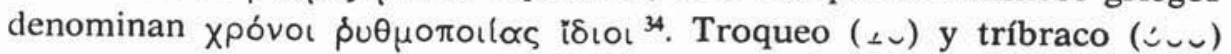
son el mismo ritmo (arsis y tesis son en ambos las mismas y con la misma relación proporcional y temporal), pero son dos figuras distintas de la ritmopea.

Estamos, por tanto, ante un mismo s is te ma que presenta unas variantes en su realización, ante una misma estructura profunda a la que corresponden varias estructuras de su. perficie.

2.1. Y es esta distinción entre $\chi \rho \delta \delta$ o

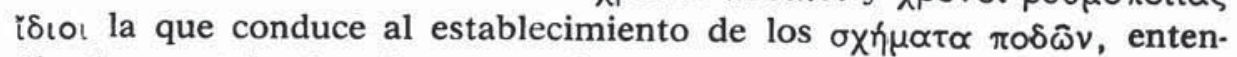
diendo por tales las diversas variantes que puede presentar un patrón rítmico-métrico determinado, o sea, empleando la terminología del sistema de niveles que presentábamos al principio, las diversas ESTRUCtuRAS de una determinada FORMA.

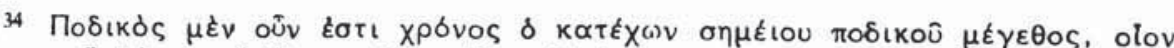

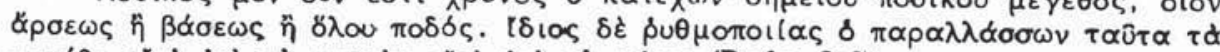

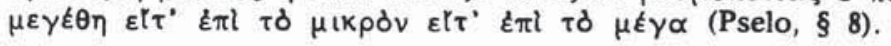

LII, $10^{\circ}-4$ 
Tales posibilidades de diversificación de una misma FORMA en distintas ESTRUCTURAS o oxń $\mu \alpha \tau \alpha$ se aprecia tanto a nivel de pie o compás

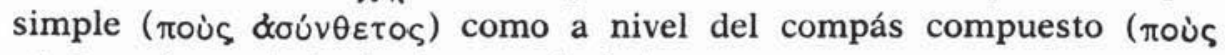
$\sigma u ́ v \theta \varepsilon \tau o \varsigma)$, es decir, incluso a nivel de verso.

2.1.1. Entre los rasgos que definen y caracterizan un pie o compás

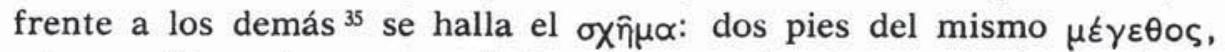

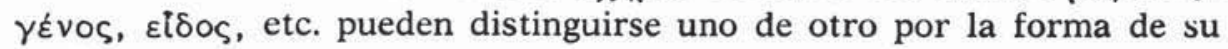
realización silábica: _ _ y y _

Se trata, pues, en el $\sigma \times \hat{\eta} \mu \alpha$ del modo y manera en que un patrón rítmico se realiza en la ritmopea mediante los elementos de un rhythmi-

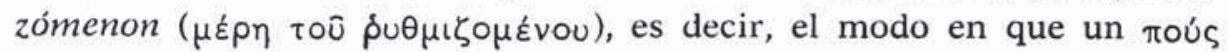

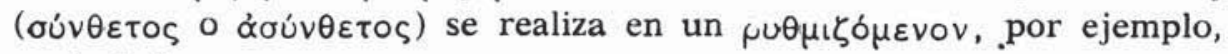
en la $\lambda \varepsilon \xi$ เs.

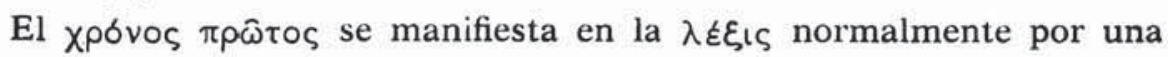

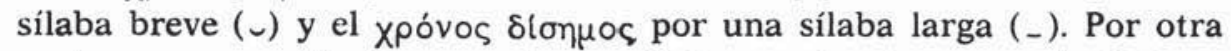
parte, como resulta lo normal que el tiempo fuerte, marcado (ßók, s,

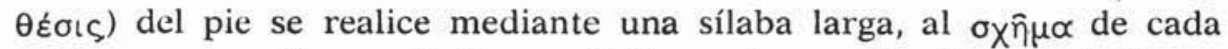
Toús que cumplía con dicha condición se lo consideró como la figura

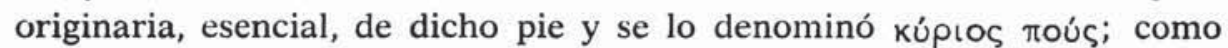
además los distintos metros recibieron muchas veces el nombre a partir de los nombres de estas figuras básicas, se los denominó también $\pi \delta \delta \delta \varsigma \mu \varepsilon \tau \rho ı o^{36}$.

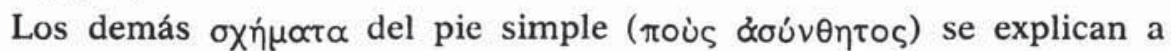

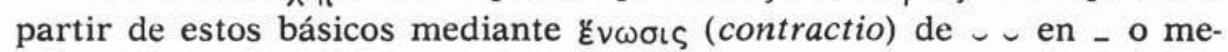

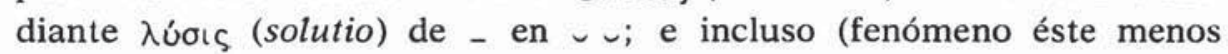

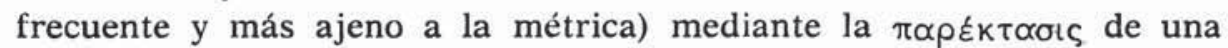
sílaba larga en otra de más de dos tiempos (\lrcorner$, \sqcup$, etc.).

Esto es lo que permite, por ejemplo, que un pie (forma o patrón

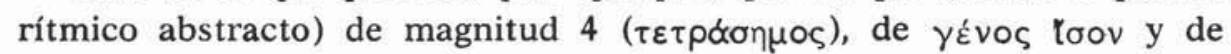

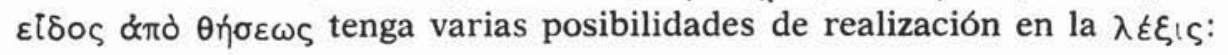

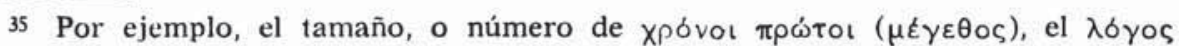
(que, según hemos visto, determina el $\gamma \varepsilon v \circ \varsigma$ ), la $\delta\llcorner\alpha(p \varepsilon \sigma ı \varsigma$ (o distinto estableci-

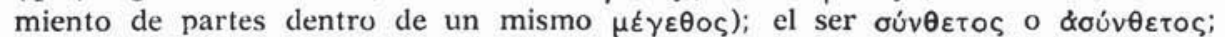

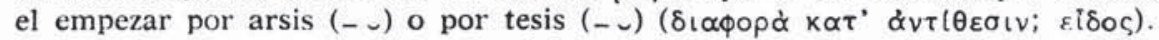

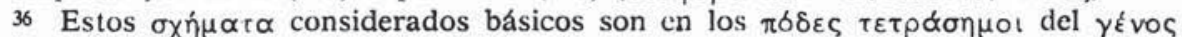

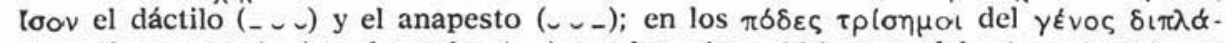

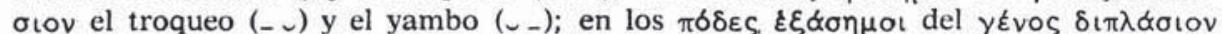
el jónico «a maiore» (___ - ) y el jónico «a minore» (_ _-_); en los $\pi \delta \delta \varepsilon \varepsilon \varsigma \pi \varepsilon v \tau \alpha$ -

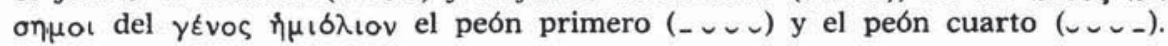




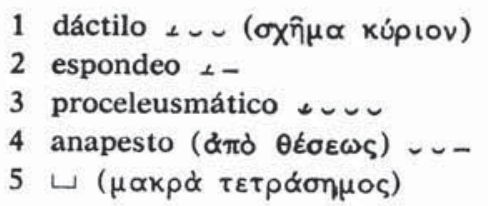

2.1.2. Las posibilidades de variación que presentan las unidades rítmicas superiores, los $\pi \delta \delta \delta \varepsilon \varsigma$ oúv $\theta \varepsilon \tau o l$, los versos, responden al mismo principio que las anteriores y, en parte, son las mismas.

Ante todo, estos $\pi \delta \delta \delta \varepsilon \varsigma$ oúv $\theta \varepsilon \tau o \iota$ pueden presentar en su realización

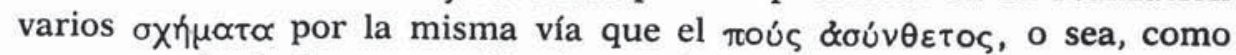

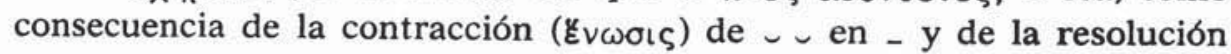

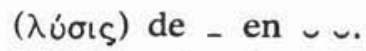

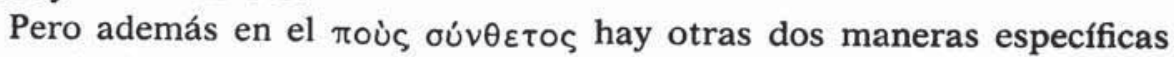

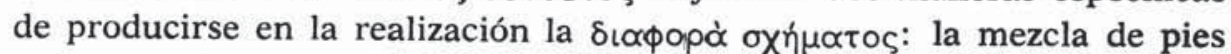

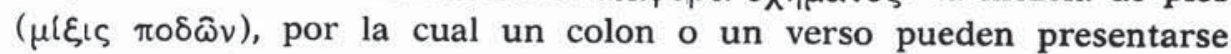

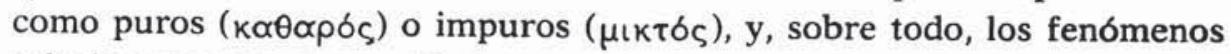
relacionados con la $\kappa \propto \tau \alpha \dot{\lambda} \eta \eta \xi เ \varsigma$.

En virtud de esto último un colon o un verso puede aparecer en la

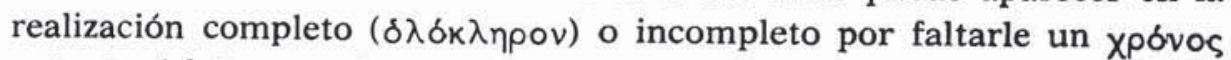

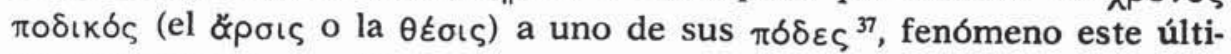
mo denominado $\kappa \propto \tau \dot{\alpha} \lambda \eta \xi\llcorner\varsigma$.

Pues bien, a efectos rítmicos, la diferencia entre acataléctico y cataléctico es en el fondo la misma que, por ejemplo, la diferencia entre

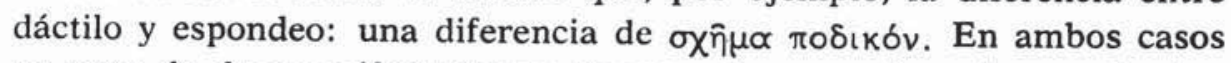
se trata de dos manifestaciones diversas de una misma forma rítmicométrica, de dos estructuras superficiales que corresponden a la misma estructura profunda.

Si Aristóxeno no nombraba, según parece, entre los cola que pueden

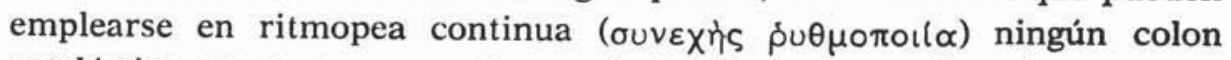

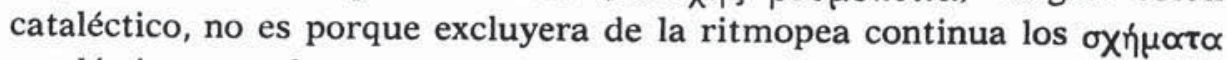
catalécticos, yendo en contra de toda la praxis de los poetas que docu-

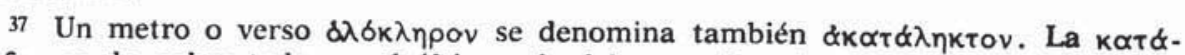

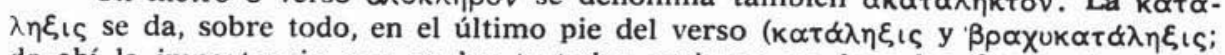
de ahí la importancia que en los tratados antiguos se da a los finales de verso),

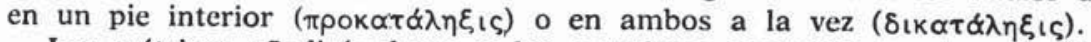

Los métricos añadirán luego a las anteriores posibilidades de variación estruc-

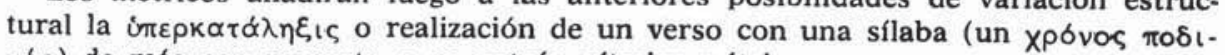
кós) de más con respecto a su patrón rítmico-métrico.

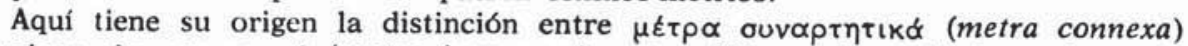

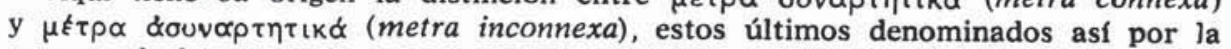
ruptura de la secuencia rítmica que produce en su interior la falta de un xpóvos

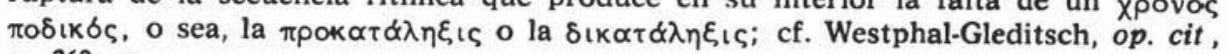
p. 260 ss. 
menta sobradamente lo contrario, sino porque para él a nivel de ritmo, que es donde se movía, no había diferencia entre cataléctico y acata-

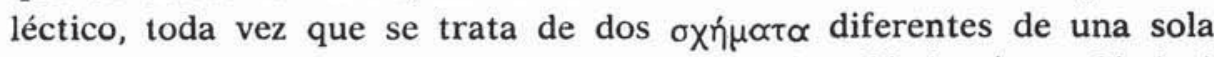
FORMA y en ese caso la diferencia entre ellos (cataléctico / acataléctico) no existe a nivel de ritmo, sino sólo a nivel de ritmopea ${ }^{38}$.

3. Así, pues, la distinción FORMA / ESTRUCTURA que, recogiéndola de los modernos tratadistas de métrica, presentábamos en nuestro sistema de niveles de análisis del lenguaje versificado se halla claramente desarrollada en la doctrina rítmica de la Antigüedad, implícitamente en la distinción entre ritmo y ritmopea e, incluso, explícitamente en la teoría

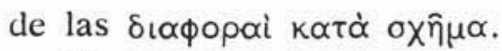

Otro tanto cabe decir acerca de la diferenciación que proponíamos entre FORMA EXTRALINGÜfSTICA O RITMICA Y FORMA MÉTRICA, distinción esencial para comprender a fondo el fenómeno lingüístico del metro y del verso en general y particularmente rentable en la métrica de las lenguas clásicas, cuyas formas rítmicas y de versificación difícilmente se pueden abordar sin tener en cuenta que nacieron en un contexto en que poesía, música y danza constituían un todo unitario.

$\mathrm{Y}$ a estos argumentos de índole técnica e histórica se añade el argumento de autoridad de que precisamente en las teorías de tratadistas de rítmica como Aristóxeno, que recogen sin duda una doctrina que se había ido conformando paulatinamente en las escuelas de artes musicales durante la época (siglos vi y v a. C.) en que aún estaban en período de gestación muchas de las formas rítmico-métricas que luego se hicieron canónicas; en una teoría que, por otra parte, pudo y debió gestarse inductivamente, a partir de la observación in uiuo de los hechos, se reconoce implícitamente dicha distinción entre FORMA RfTMICA (extralingüística) y FORMA MÉTRICA, toda vez que se distingue entre xpó-

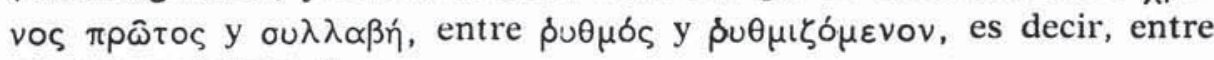
rítmica y métrica ${ }^{39}$.

Tal distinción por parte de Aristóxeno supone con respecto al pasado una superación de lo que hasta entonces parece que había sido confusión y dependencia excesiva de aquélla con respecto a ésta. Pero, a la vez, respecto al futuro, significa no sólo un sabio discernimiento de dos niveles de suyo distintos, sino el mantenimiento en su justo punto de unas relaciones innegables entre ambos.

38 Cf. Westphal-Gleditsch, op. cit., p. 171

39 En este sentido Aristóxeno es para nosotros el primero de los $x \omega \rho / \zeta o v \tau \varepsilon \varsigma$, la escuela postaristoxénica de rítmicos, denominada así por Aristides Quintiliano porque operaban con una distinción metódica entre rítmica y métrica. 
Quizá fue el propio desarrollo histórico, tanto de la praxis métrica como, y sobre todo, de los condicionamientos a que se vio sometido el estudio de la métrica desde época alejandrina, lo que motivó que este difícil equilibrio no supieran mantenerlo los metricólogos de épocas posteriores. Condicionada la métrica posterior por la gramática y por la labor filológica, fue olvidando cada vez más sus verdaderos lazos de unión con la rítmica y o bien llegó a cortar por completo el cordón umbilical que la unía con su madre nutricia o bien acudió esporádicamente a beber sin tino y sin mesura en sus fuentes; extremos ambos a cuál más dañino para la teoría del metro tal y como se fue transmitiendo durante siglos.

De la mano de la lingüística contemporánea la métrica, clarificando su ámbito y su cometido, ha sabido empezar a despojarse de muchas de las adherencias que había ido recogiendo a lo largo de tanto tiempo $y$, aunque partiendo de perspectivas y de premisas distintas, vuelve a ocupar las posiciones que, al parecer, un día tuvo. El que dichas posiciones, como acabamos de ver, sean en algunos aspectos las mismas que hace veinticuatro siglos, el que se hagan hoy formulaciones teóricas que coinciden, si no en la forma, sí en el fondo con las que ya habían hecho los tratadistas griegos de cuatro siglos antes de Cristo, no debe llevarnos a un sentimiento de frustración presidido por la idea del nihil nouum sub sole, sino, al contrario, debe ser valorado como un logro, si es que, como parece ser, verdaderamente en esos casos los caminos seguidos por los estudiosos de hoy se encuentran con los que siguieron los de entonces.

Y ello, sobre todo, cuando se trata de una misma doctrina formulada dos veces desde presupuestos distintos y, más aún, cuando para el enunciado de hoy es sin duda una garantía coincidir con lo que dijeron quienes fueron testigos y observadores directos de los hechos.

Siendo así todas las coincidencias de que hemos hablado un auténtico aval que legitima la distinción de todos los anteriores niveles dentro del fenómeno métrico, merece incluso la pena recoger en las formulaciones actuales de la teoría algunos de los términos que dentro de dicha teoria fueron ya empleados por los antiguos.

Según eso, de acuerdo con lo dicho más arriba, para el primero de los niveles en cuestión la denominación FORMA RfTMICA parece preferible a la de Forma eXtralingǘfstica. Y, sobre todo, parece conveniente sustituir en el nivel tercero el nombre de estructura por el de Esoubma. 
Semejante cambio en la terminología reflejaría de algún modo el reconocimiento de que la moderna teoría coincide en buena parte con las doctrinas de la antigua rítmica y corrobora los que ya entonces

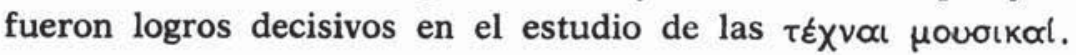

Jesús LuQue MORENo 